Submitted to the:

2003 Particle Accelerator Conference

$\mathrm{BNL}-69592-\mathrm{CP}$

Portland, Oregon, May 12-16, 2003

\title{
DESIGN OF A FAST CHROMATICITY JUMP IN RHIC *
}

\author{
C. Montag ${ }^{\dagger}$, J. Kewisch, D. Bruno, G. Ganetis, W. Louie, BNL, Upton, NY 11973, USA
}

\section{Abstract}

During transition crossing in the Relativistic Heavy Ion Collider (RHIC), chromaticities have to change sign. This sign change is partially accomplished by the $\gamma_{t}$ quadrupole jump; however, the resulting chromaticity jump is only $\Delta \xi_{x}=2.1$ in the horizontal and $\Delta \xi_{y}=2.4$ in the vertical plane. To increase the jump height, a dedicated chromaticity jump scheme has been designed, consisting of fast power supplies connected to six sextupoles per ring, which is capable of providing a chromaticity jump of $\Delta \xi=6$.

\section{INTRODUCTION}

The Relativistic Heavy Ion Collider (RHIC) consists of two superconducting storage rings installed side-by-side, which intersect at six interaction points. Therefore, each ring consists of three inner and three outer arcs. Ions other than protons are injected into RHIC from the Alternating Gradient Synchrotron (AGS) below transition energy, at $\gamma$ in the range of 10 to 15 . When accelerated to storage energies of $100 \mathrm{GeV} /$ nucleon, which corresponds to $\gamma=107$ for fully stripped gold ions, for example, the transition energy has to be crossed at $\gamma_{t}=23.2$. This is accomplished by a dedicated set of $\gamma_{t}$-quadrupoles which are equipped with special power supplies capable of switching the sign of the magnetic field within some $30 \mathrm{msec}$.

This sign flip modifies the horizontal dispersion $D_{x}$, leading to a change in the transition energy as

$$
\alpha=\frac{1}{\gamma_{t}^{2}}=\frac{1}{C} \int_{0}^{C} \frac{D_{x}(s)}{\rho(s)} \mathrm{d} s,
$$

where $C$ is the circumference of the ring, and $\rho(s)$ designates the local bending radius. Figure 1 shows the resulting horizontal dispersion function $D_{x}$ before and after the transition jump.

Since the arcs are equipped with regular sextupoles to control the chromaticities of the machine, the modified dispersion function within these sextupoles slightly changes the chromaticities. In the case of RHIC, the associated chromaticity change due to the $\gamma_{t}$-quadrupole sign change is $\Delta \xi_{x}=2.1$ horizontally and $\Delta \xi_{y}=2.4$ vertically for a $\gamma_{t}$ jump height of $\Delta \gamma_{t}=1.0$.

The positive sign of this chromaticity change provides the necessary sign flip when crossing transiton. However, operational experience shows that the magnitude of this chromaticity jump may not be sufficient, because chromaticites have to be kept around $\xi_{x, y}=-1$ before transition to ensure a symmetric jump with $\xi_{x, y}=+1$ after $\gamma_{t}$. Keeping

\footnotetext{
${ }^{*}$ Work performed under contract number DE-AC02-98CH10886 with the auspices of the US Department of Energy

† montag@bnl.gov
}
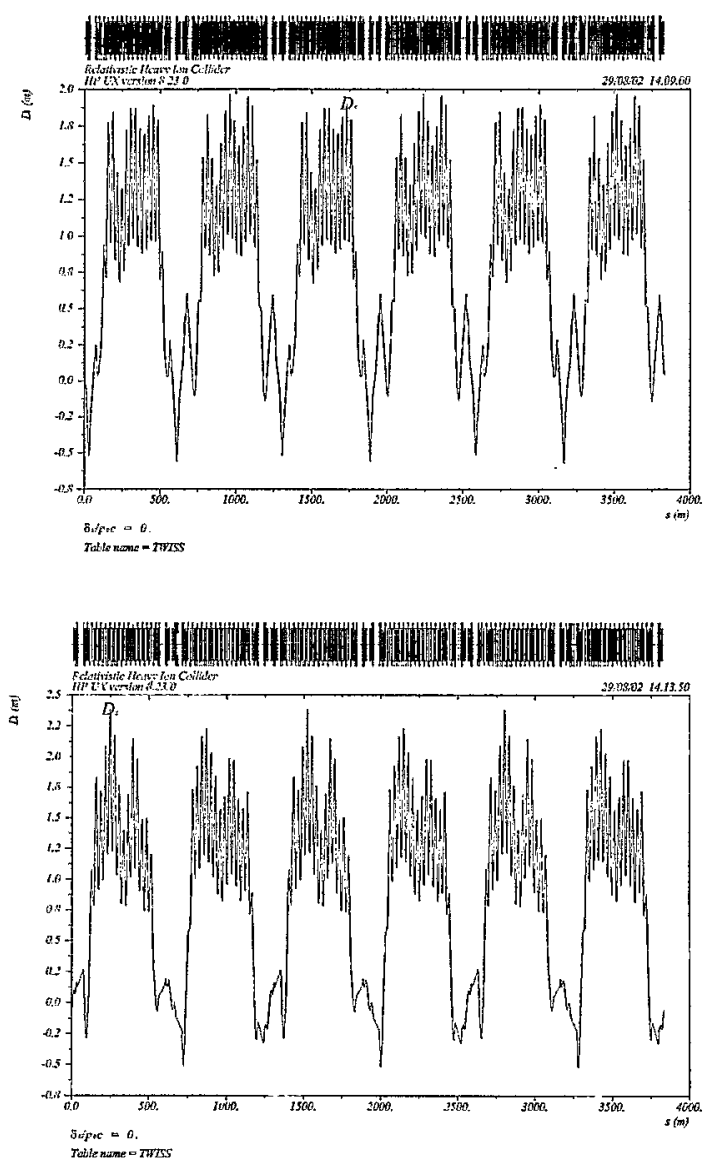

Figure 1: Horizontal dispersion function $D_{x}$ before (top) and after (bottom) crossing transition for $\Delta \gamma_{t}=1.0$.

chromaticites that close to zero can lead to transverse instabilites around transition, as it was observed during RHIC operations.

To improve this situation, a dedicated chromaticity jump scheme was developed [1]. This scheme consists of two sextupoles at one end of each outer RHIC arc, which are jumped from zero to their final strength within $30 \mathrm{msec}$ at transition. Since this leads to an uneven sextupole distribution around the ring, we performed extensive dynamic aperture studies to ensure the feasibility of this scheme. This paper presents the results of these studies. 


\section{RESULTS}

Dynamic apertures where calculated by MAD [2] for three different values of $\Delta p / p:-0.002,0.0$, and +0.002 . At injection and transition, $\Delta p / p=0.002$ corresponds to roughly one $\sigma_{p}$, while it corresponds to about two $\sigma_{p}$ at store. Tunes were set to $Q_{x}=28.21$ horizontally and $Q_{y}=29.23$ vertically, and the $b_{2}$ component of the dipoles was included in the tracking according to magnetic field measurements [3].

At injection with a beta-function of $\beta_{x, y}^{*}=10 \mathrm{~m}$ at all IPs the jump sextupoles are off, as well as the $\gamma_{t}$-quadrupoles. The remaining regular arc sextupoles correct the chromaticities in both planes to $\xi_{x, y}=-2$. Figure 2 shows the resulting dynamic aperture in the $(x-y)$ plane, together with the result for the existing regular sextupole scheme. The two schemes do not differ significantly.

Right before the transition jump, the jump sextupoles are
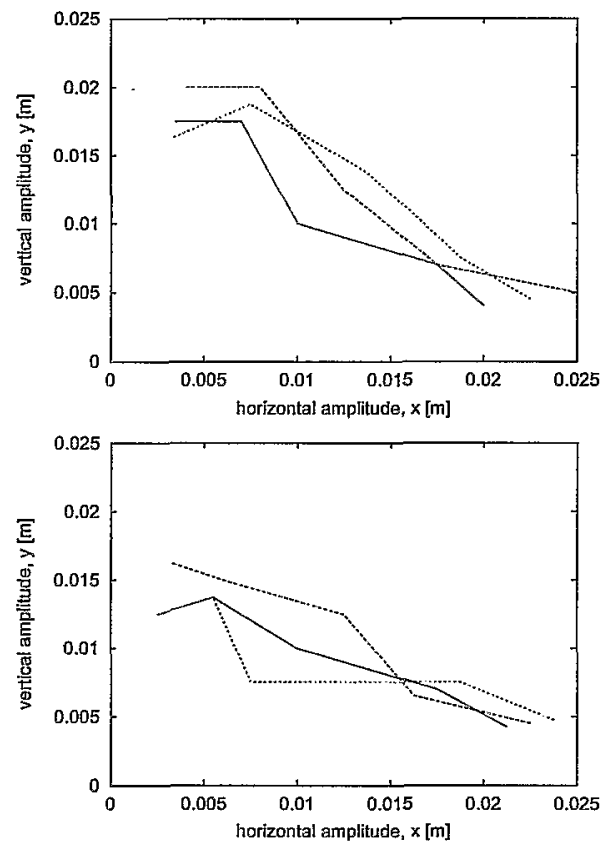

Figure 2: Dynamic aperture at injection in the $(x-y)$ plan $e$, for $\Delta p / p=0.0$ (solid), 0.002 (dashed) and -0.002 (dotted). The $\beta$-function in all IPs is set to $\beta^{*}=10 \mathrm{~m}$. One $\sigma$ corresponds to $1.25 \mathrm{~mm}$ in both planes for an emittance of $\epsilon=10 \pi \mathrm{mm}$ mrad. The top graph shows the situation with the new jump sextupole configuration, while the lower plot exhibits the dynamic aperture for the regular sextupole scheme.

still off, and the remaining regular arc sextupoles provide chromaticities of $\xi_{x, y}=-2$ in both planes. Additionally, the $\gamma_{t}$-quadrupoles are on and provide an increased $g a m m a_{t}$ value, with $\Delta \gamma_{t}=0.5$. The resulting dynamic aperture is significantly smaller than for the regular scheme (see Figure 3), but since it is still at least $12 \sigma_{x, y}$ it is con- sidered sufficient.

Right after the transition jump, the $\gamma_{t}$-quadrupoles have
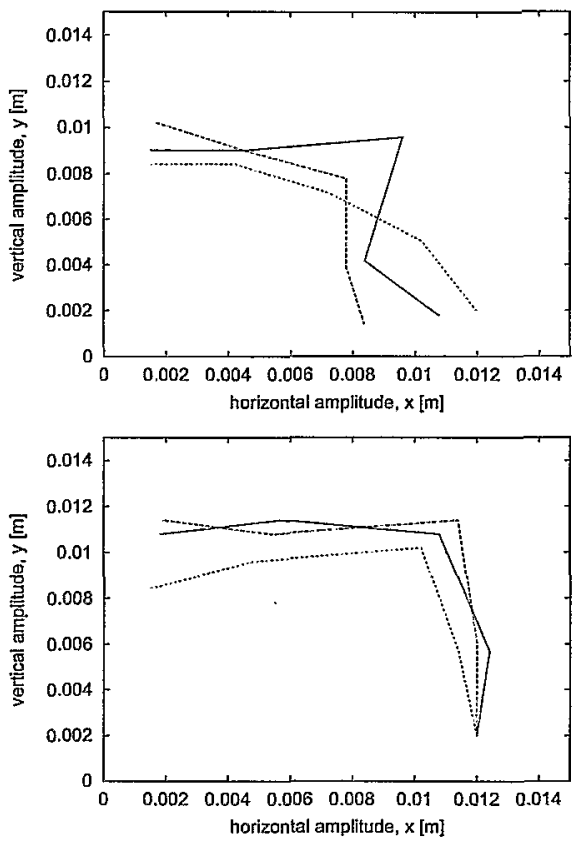

Figure 3: Dynamic aperture just before the transition jump, for $\Delta p / p=0.0$ (solid), 0.002 (dashed) and -0.002 (dotted). The $\beta$-function in all IPs is $\beta^{*}=5 \mathrm{~m}$. One $\sigma$ corresponds to $0.6 \mathrm{~mm}$ in both planes for an emittance of $\epsilon=10 \pi \mathrm{mm}$ mrad. The top graph shows the situation with the new jump sextupole scheme, while the lower plot depicts the dynamic aperture for the regular scheme.

flipped sign. The jump sextupoles are turned on now and set the total chromaticities to $\xi_{x, y}=+2$, while the strength of the remaining regular arc sextupoles remains unchanged. This results in a dynamic aperture that is practically the same as for the regular sextupole scheme, Figure 4.

At store the $\gamma_{t}$ quadrupoles are off again, while the jump sextupoles are set to the maximum strength the fast power supplies are capable of providing, which corrresponds to roughly the same strength as the remaining sextupoles in the horizontal plane and about half of the regular sextupole strength vertically. The resulting dynamic aperture for a $\beta$ function of $\beta_{x, y}^{*}=2 \mathrm{~m}$ at all IPs is shown in Figure 5. As a comparison with the existing regular sextupole scheme shows, the dynamic aperture is practically the same for both configurations.

\section{CONCLUSION}

Dynamic aperture studies have been performed to ensure the feasibility of a fast chromaticity jump to improve transition crossing performance in RHIC. Comparison with the existing regular sextupole configuration showed suffcient dynamic aperture in spite of the resulting irregular 

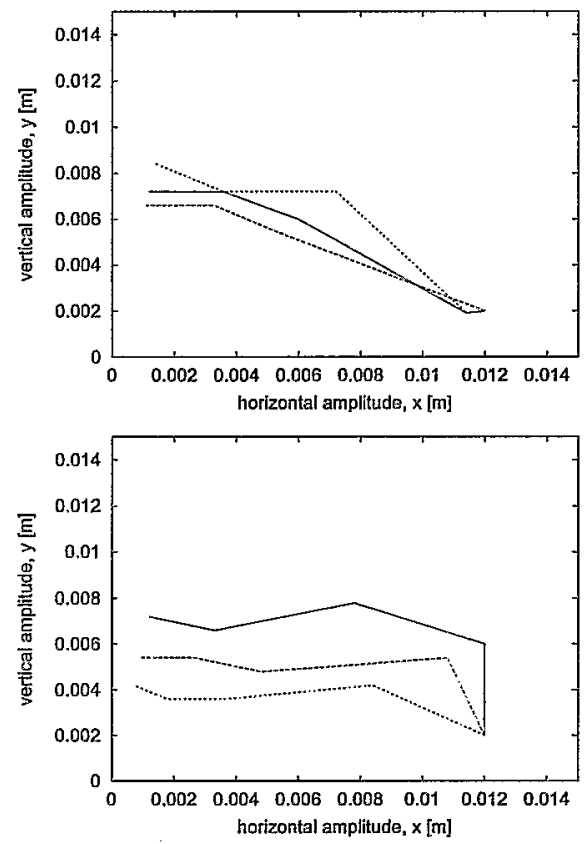

Figure 4: Dynamic aperture just after the transition jump, f or $\Delta p / p=0.0$ (solid), 0.002 (dashed) and -0.002 (dotted), for $\beta^{*}=5 \mathrm{~m}$ in all IPs. One $\sigma$ corresponds to $0.6 \mathrm{~mm}$ in both planes for an emittance of $\epsilon=10 \pi \mathrm{mm}$ mrad. The top graph shows the situation with the jump sextupoles, while the lower plot exhibits the dynamic aperture for the regular scheme.

sextupole distribution. This scheme will therefore be implemented for the upcoming RHIC run.

\section{REFERENCES}

[1] C. Montag, J. Kewisch, Dynamic aperture studies towards a $\gamma_{t}$ sextupole jump in RHIC, BNL C-A/AP/84

[2] H. Grote, F. C. Iselin, The MAD program, CERN/SL/90-13 (AP)

[3] S. Tepikian, private communication
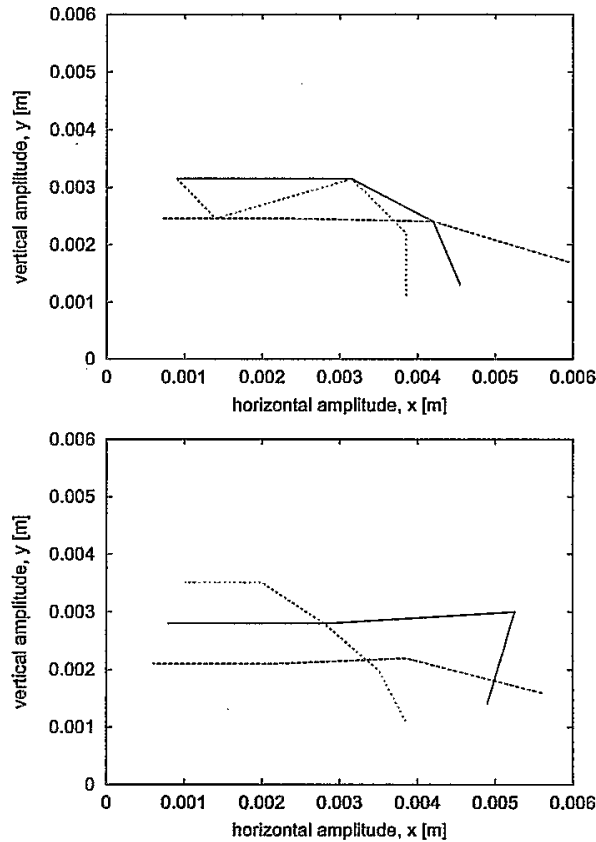

Figure 5: Dynamic aperture at store, for $\Delta p / p=0.0$ (solid), 0.002 (dashed) and -0.002 (dotted), for $\beta^{*}=$ $2 \mathrm{~m}$ in all IPs. The jump sextupoles are ON, providing $87 \%$ of the regular sextupole strength in the horizontal direction, and $44 \%$ in the vertical plane. One $\sigma$ corresponds to $0.35 \mathrm{~mm}$ in both planes for an emittance of $\epsilon=40 \pi \mathrm{mm} \mathrm{mrad}$ (end of store). The top graph depicts the situation with jump sextupole configuration, while the lower plot shows the dynamic aperture for the regular sextupole scheme. Note that at the end of the store, the emittance is assumed to be blown up to $\gamma \epsilon=40 \pi \mathrm{mm}$ mrad, resulting in an rms beam size of $0.35 \mathrm{~mm}$ at the $\mathrm{IP}$. 